\title{
Krüppel-like factor 10 null mice exhibit lower tumor incidence and suppressed cellular proliferation activity following chemically induced liver tumorigenesis
}

\author{
SEUNG-HO HEO ${ }^{1,2}$, EUI-SUK JEONG ${ }^{1}$, KYOUNG-SUN LEE ${ }^{3}$, JIN-HEE SEO ${ }^{4}$, \\ WOON-KYU LEE ${ }^{5}$ and YANG-KYU CHOI ${ }^{1}$
}

\footnotetext{
${ }^{1}$ Department of Laboratory Animal Medicine, College of Veterinary Medicine, Konkuk University, Seoul 143-701;

${ }^{2}$ Asan Institute for Life Sciences, University of Ulsan College of Medicine, Seoul 138-736;

${ }^{3}$ Laboratory Animal Center, Osong Medical Innovation Foundation, Chungbuk 363-951;

${ }^{4}$ Laboratory Animal Facility, Korea Institute of Radiological and Medical Sciences, Seoul 139-706;

${ }^{5}$ Laboratory of Developmental Genetics, College of Medicine, Inha University, Incheon 402-751, Republic of Korea
}

Received October 27, 2014; Accepted January 16, 2015

DOI: $10.3892 / o r .2015 .3801$

\begin{abstract}
Liver cancer is the third most common cancer, and the incidence as well as the mortality rate of liver cancer are on the increase. There are many signaling pathways that are involved in hepatic tumorigenesis. One of these pathways, the transforming growth factor- $\beta$ (TGF- $\beta$ )/Smad pathway with KLF10, has been reported to suppress cellular proliferation in most cases. However, the actual functions of KLF10 in various pathophysiological conditions are still fragmentary and unclear. In the present study, the practical role of KLF10 in DEN-induced hepatic carcinogenesis, was elucidated using KLF10 null mice. In the necropsy and histopathological analysis, KLF10 KO mice exhibited lower tumor incidence and PCNA labeling indices than these values in the wild-type mice. Additional analyses revealed that the mRNA and protein levels of Smad3, TGF- $\beta 1$, TGF- $\beta$ RI and p15 were increased in the tumor tissues of the KLF10 KO mice, while those of cMyc and cyclin D1 were downregulated. The level of phospho-Smad3 was also significantly higher in the tumor tissues of the KLF10 KO mice. All together, the KLF10 KO condition may reinforce the TGF- $\beta$-Smad signaling pathway and confer tumor-suppressor effects against chemically induced liver tumorigenesis.
\end{abstract}

\section{Introduction}

Liver cancer is a major public health issue, and the incidence as well as the mortality of liver cancer are on the increase.

Correspondence to: Professor Yang-Kyu Choi, Department of Laboratory Animal Medicine, College of Veterinary Medicine, Konkuk University, 1 Hwayang-dong, Gwangjin-gu, Seoul 143-701, Republic of Korea

E-mail: yangkyuc@konkuk.ac.kr

Key words: KLF10, TGF- $\beta$, hepatic tumorigenesis, antiproliferation
Globally, new cases of liver cancer have been annually reported to be $\sim 700,000$, and East Asian countries, including Korea, Japan and China, are considered to be high-risk areas (1-3). The risk factors for liver cancer include chronic hepatitis virus, dietary contaminants, fatty liver diseases, cirrhosis and host genetic factors $(4,5)$. Many signaling pathways are also involved in hepatic tumorigenesis, including various growth factors such as the Wnt, Hedgehog and TGF- $\beta$ pathway (6-8).

Transforming growth factor- $\beta$ (TGF- $\beta$ ) is a secreted cytokine that regulates many biological processes in most cells. It performs important functions in early and late embryogenesis, and also plays a key role in adult tissues, including cellular proliferation, apoptosis, differentiation, angiogenesis, extracellular matrix synthesis and immune responses $(9,10)$. According to the cell or tissue type and the microenvironment, the cellular responses to TGF- $\beta$ are different. Various target genes, including collagen type I, $\alpha$-smooth muscle actin, SNAI1 and p15, may be induced in the liver after activation of the TGF- $\beta$ pathway. These genes may induce diverse responses, including fibrosis, cellular migration and suppression of cellular proliferation $(11,12)$. Several studies have shown that the TGF- $\beta$ pathway may suppress cellular proliferation, including early tumorigenesis and liver regeneration in most cases. However, the role of TGF- $\beta$ in various pathological conditions is still unclear (13-15).

The TGF- $\beta /$ Smad pathway initiates with activation of secreted TGF- $\beta$ and the ligand assembles TGF- $\beta$ receptor type I and II (TGF- $\beta$ RI and II) in the cellular membrane. Then, the TGF- $\beta$ RII phosphorylates and activates the TGF- $\beta$ RI. Smad2 (ser465/476) and Smad3 (ser433/435) are then phosphorylated by activating TGF- $\beta$ RI and are also called receptor-Smads (R-Smads). Activated R-Smads bind Smad4, also referred to as common Smads (Co-Smads), and pass the nuclear membrane. In the nucleus, the Smad complex binds with co-factors and positively and negatively regulates the expression of target genes $(10,13,16,17)$. The expression levels of p15, p21, KLF10 and Smad 2 genes are also increased and Smad7, cMyc, cyclin D1 
genes or proteins are downregulated in the proliferation control by the TGF- $\beta /$ Smad pathway $(8,10)$. In the non-Smad TGF- $\beta$ pathway, TGF- $\beta$ RI may also activate other signaling systems, including PI3K, RAS and FAS (9,17-19).

KLF10 is an early target gene of the TGF- $\beta /$ Smad (Smaand Mad-related) signaling pathway and it is reported that it may inhibit cellular proliferation in various cell types $(20,21)$. At first, KLF10 was observed in normal human osteoblasts after TGF- $\beta$ treatment. Thus, KLF10 was initially named as TGF- $\beta$ inducible early gene-1 (TIEG1) (22). Later, considering three zinc finger domains of the TIEG1 protein, it was classified and renamed as Krüppel-like family of transcription factors 10 (20,21,23). Recent studies have shown that KLF10 expression may activate the TGF- $\beta /$ Smad pathway and mimic the roles of TGF- $\beta$ in several cell types. KLF10 may enhance the transcription of TGF- $\beta$-regulated genes, including Smad2, p21, TGF- $\beta 1$ and plasminogen activator inhibitor-1 and suppress the transcription of the Smad7 gene, inhibitory Smads $(13,24,25)$. However, there has been limited information concerning the role of KLF10.

KLF10, one of the target genes of TGF- $\beta$, plays an important role in numerous biological processes, including tumorigenesis. However, there has been limited information concerning KLF10, and the actual functions of KLF10 in liver tumorigenesis are still unknown. In the present study, to elucidate the role of KLF10 in chemically induced hepatic carcinogenesis, histopathological and molecular analyses were performed using KLF10 KO mice injected with diethylnitrosamine (DEN) via intraperitoneal route.

\section{Materials and methods}

Mice. Male KLF10-deficient C57BL/6J mice were kindly provided by Dr Woon-Kyu Lee (Inha University, Incheon, Korea) (26), and the C57BL/6J mice were obtained from the Korea Research Institute of Bioscience and Biotechnology (KRIBB, Daejeon, Korea). All mice were bred in a laboratory animal breeding room under specific pathogen-free conditions. For genotyping, the DNA samples were isolated from all mouse tails using a genomic DNA extraction kit (Bioneer, Daejeon, Korea) and subjected to polymerase chain reaction. The DNA primers for genotyping were KLF10 F, CCTTCCTGCCAACAACTCTC; R, TCTGAGGAGGAC CCTTGCT and KLF10 KO F, TCGCCTTCTTGACGAG TTCT. The size of the KLF10 KO gene is 658 base pairs (bp) and that of the wild-type (WT) gene is $248 \mathrm{bp}$. All the procedures were approved by the IACUC (Institutional Animal Care and Use Committee, KU 09023, Seoul, Korea) of Konkuk University.

Experimental design. For the liver carcinogenesis study, $25 \mathrm{mg} / \mathrm{kg}$ of DEN was single injected in the mice via intraperitoneal route at 12 days of age. KLF10 KO and WT mice (13-14 mice/group) were sacrificed at 26 and 36 weeks, respectively, after the carcinogen administration (at the age of 28 and 38 weeks, respectively). Three mice injected with PBS were used as control animals in each group. At the time of the sacrifice, all the mice were grossly examined, and blood was collected from the caudal vena cava. The liver was excised and weighed. Some portion of the liver tissue was fixed in $10 \%$ neutral-buffered formalin and the rest of the hepatic tissue was frozen for further analysis.

Hematoxylin and eosin (H\&E) staining and histopathological analysis. Fixed liver tissues were processed by standard methods, embedded in paraffin and then cut in 4- $\mu$ m sections. The sections were deparaffinized, rehydrated and stained with H\&E. Furthermore, the sections were dehydrated, cleared, mounted and viewed by light microscopy. The hepatic lesions were classified as hepatic foci, hepatocellular adenoma (HCA) or hepatocellular carcinoma (HCC). Two veterinary pathologists independently reviewed all the lesions.

Immunohistochemical staining. For immunohistochemistry, some serial sections $(4-\mu \mathrm{m})$ were deparaffinized, rehydrated, placed in $0.01 \mathrm{M}$ citrate buffer $(\mathrm{pH}$ 6.0) and heated in a microwave for $10 \mathrm{~min}$. Then, the slides were incubated for $10 \mathrm{~min}$ in $1.0 \% \mathrm{H}_{2} \mathrm{O}_{2}$. The slides were preincubated with blocking serum (Vectastain ABC kit; Vector Laboratories, Burlingame, CA, USA), incubated with proliferative cell nuclear antigen (PCNA; Santa Cruz Biotechnology Inc., Santa Cruz, CA, USA) antibody and then incubated with biotinylated secondary antibodies (Vectastain ABC kit) followed by incubation with avidin-coupled peroxidase (Vectastain ABC kit) (both from Vector Laboratories). After development with 3,3'-diaminobenzidine (DAB, DAB Substrate kit; Vector Laboratories) the sections were counterstained with hematoxylin.

Quantitative reverse transcription-polymerase chain reaction (RT-PCR) analyses. RT-PCR was performed on RNA samples that were isolated from the liver tissues. Total RNA was prepared from frozen liver tissues using TRIzol (Invitrogen) and reverse transcribed into cDNA using M-MLV reverse transcriptase (both from Invitrogen, Carlsbad, CA, USA). The cDNA was used as a template for amplification in the PCR. Band intensities were quantified using the Multi Gauge software V3.0 (Fujifilm, Tokyo, Japan) and normalized to transcription levels of $\beta$-actin. The sequences of the primer pairs were as follows: Smad2 F, CGGTTAGATGAGCTTGA GAA and R, TCACTGATATATCCAGGTGG; Smad3 F, TCACTGGATGGTCGGCTGCA and R, CTTCACTCAGGT AGCCAGGA; Smad4 F, CGATGCCTGTCTGAGCATTG and R, CTCCGTTGATGCGCGATTAC; Smad7 F, ATCGGT CACACTGGTGCGTG and R, TCCAGTGTGGCGGACTT GAT; p15 F, CTGGATGTGTGTGACGCCTG and R, CTT CGTGCTTGCAGTCTTCC; p21 F, AGCTAGGATGACAG TGAAGC and R, CAAGTGCCTAGATATGCCTC; TGF- $\beta$ RI F, TATGATATGACAACATCAGG and R, AACCACAGC TGCGTCCATGT; TGF- $\beta$ RII F, GTGTGCCTGTAACATGG AAG and R, AGGTGTTCTGCTTCAGCTTG; c-myc F, TAG TGCTGCATGAGGAGACA and R, GTTGCTGATCTGCT TCAGGA; cyclin D1 F, GGATGCTGGAGGTCTGTGAG and R, GAGTTGTCAGTGTAGATGCAC, $\beta$-actin F, TGGAAT CCTGTGGCATCA TGAAA and R, TAAAACGCAGCTCAG TAACAGTCC.

Western blot analysis. Protein was extracted from the liver with extraction solution (Pro-Prep ${ }^{\mathrm{TM}}$; Intron Biotechnology, Seoul, Korea). The protein concentrations were determined using the BCA kit (Pierce Biotechnology Inc., Rockford, IL, 


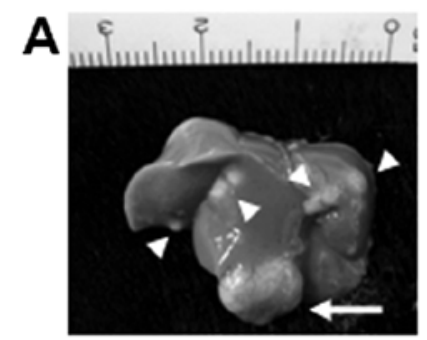

Wild-type

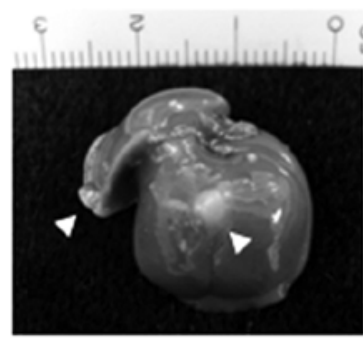

KLF10 KO
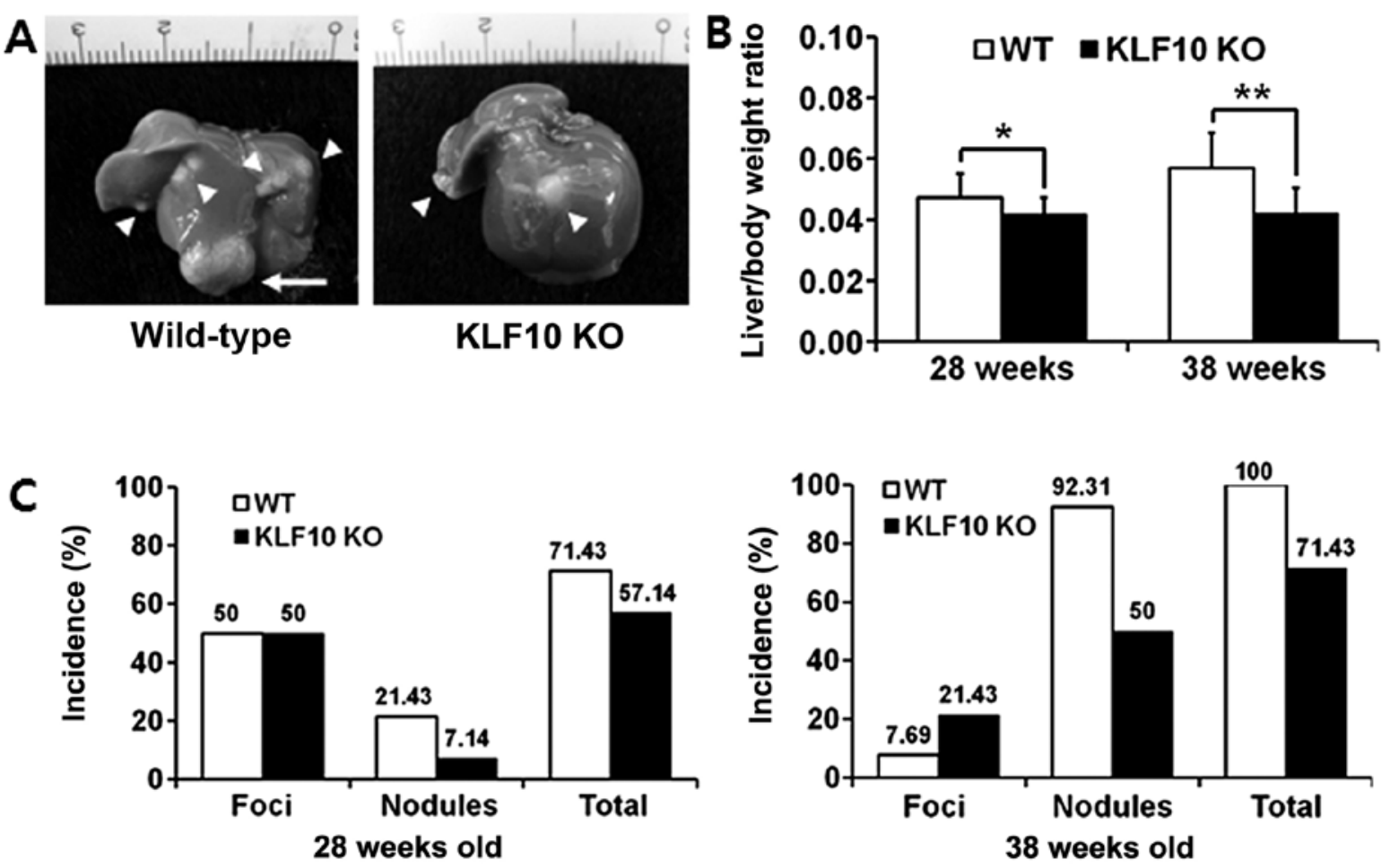

Figure 1. Development of liver tumors and necropsy findings. (A) Gross findings of excised liver. All the masses were divided into foci ( $<10$ mm) and nodules ( $\geq 10 \mathrm{~mm}$ ). KLF10 KO mice had less masses and nodules than the WT mice. Arrowheads, foci, arrow, nodule. (B) The mean liver/body weight ratio was significantly higher in the WT than the ratio in the KLF10 KO mice at both 28 and 38 weeks of age. ${ }^{*} \mathrm{P}<0.05$ and ${ }^{* *} \mathrm{P}<0.01$ by unpaired Student's t-test. $(\mathrm{C}) \mathrm{The}$ incidence of nodules and total masses was higher in the WT than the incidence in the KLF10 KO mice at both 28 and 38 weeks of age. WT, wild-type.

USA). After being transferred onto nitrocellulose membranes, the proteins were blocked and incubated overnight with specific antibodies against $\beta$-actin, Smad4, Smad7, p15, p21, TGF- $\beta$ RI, cMyc (Santa Cruz Biotechnology, Inc.), Smad2, Smad3, p-Smad3, p27, TGF- $\beta$ RII and cyclin D1 (Cell Signaling Technology Inc., Beverly, MA, USA) at $4^{\circ} \mathrm{C}$. Then, the membranes were washed with TBST and incubated with either anti-rabbit or anti-mouse secondary antibodies (Santa-Cruz Biotechnology, Inc.), which were horseradishperoxidase linked. Specific antibodies were detected with an ECL test kit (KPL, Gaithersburg, MD, USA). The band intensities were quantified using Imagequant Software (Image Lab V4.0; Bio-Rad Inc., San Diego, CA, USA) and normalized to $\beta$-actin expression.

Statistical analysis. For statistical analysis, all data obtained were analyzed using SPSS V14.0 software (SPSS Inc., Chicago, IL, USA). Statistically significant differences between the studied groups were evaluated using the unpaired Student's t-test or the Fisher's exact test. Results were determined to be statistically significant for values of $\mathrm{P}<0.05(\mathrm{P}<0.05$ and $\mathrm{P}<0.01$ are indicated in the figure legends).

\section{Results}

Gross findings and histopathological analysis. KLF10 KO mice ( $\mathrm{n}=14$ and 14, respectively) and WT mice ( $\mathrm{n}=14$ and 13, respectively) were sacrificed at 26 and 36 weeks after DEN injection (at the age of 28 and 38 weeks, respectively), and the body and liver weights were determined. The number and diameter of the masses in the liver were determined. All the masses were divided into foci $(<10 \mathrm{~mm})$ and nodules $(\geq 10 \mathrm{~mm})$ depending on their size (Fig. 1A).

The liver/body weight ratios were significantly lower in the KLF10 KO than these ratios in the WT mice. At the age of 28 weeks, the mean \pm standard deviation (SD) values of the ratios of the WT and KLF10 KO mice were 0.047 \pm 0.0079 and $0.042 \pm 0.0054$, respectively $(\mathrm{P}=0.046)$. At the age of 38 weeks, the mean \pm SD ratios of the WT and KLF10 KO mice were $0.056 \pm 0.00076$ and $0.042 \pm 0.0083$, respectively $(\mathrm{P}=0.00077)$ (Fig. 1B). The incidence of masses was also lower in the KLF10 KO than that in the WT mice. The total incidence of masses was 71.43 vs. $57.14 \%$ at 26 weeks after DEN treatment, and 100 vs. $71.43 \%$ at 36 weeks after DEN treatment in the WT and KLF10 KO mice, respectively. In addition, the WT mice had more nodules than those in the KLF10 KO mice. The total incidence of nodules was $21.43 \mathrm{vs} .7 .14 \%$ at the age of 26 weeks and 92.31 vs. $50 \%$, at the age of 36 weeks in the WT and KLF10 KO mice, respectively, after DEN treatment (Fig. 1C).

In the DEN-treated mice, the hepatic lesions progressed from hepatic foci, HCA to HCC. In the microscopic observation, the hepatic foci showed irregular cellular size. Most hepatic foci consisted primarily of clear or vacuolated cells, and partially of basophilic cells. HCAs displayed well-circumscribed lesions, composed of hepatocytes with cellular pleomorphism, anisokaryosis and basophilic or vacuolated cytoplasm containing hepatocytes. Most of the adenomas did not have normal lobular architecture and their vasculatures were compressed or altered. In HCCs, cellular atypia with malignancy, an increase in the nuclear-cytoplasmic ratio and mitotic figures were common and a trabecular growth 


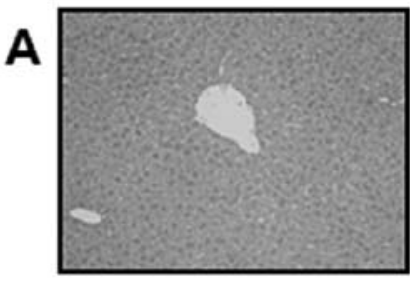

Normal

B

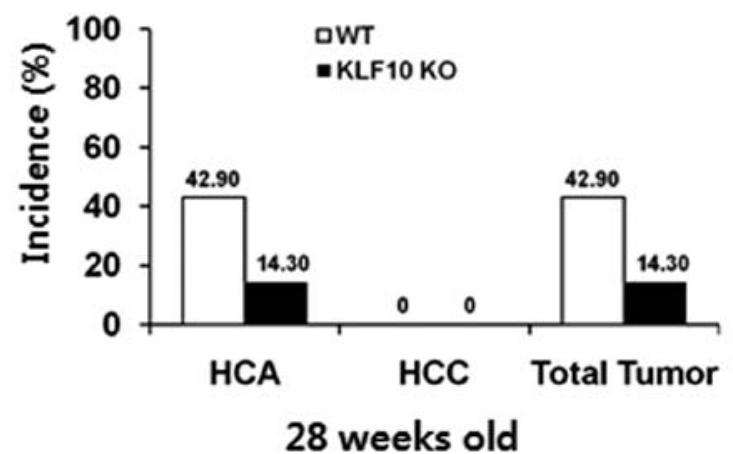

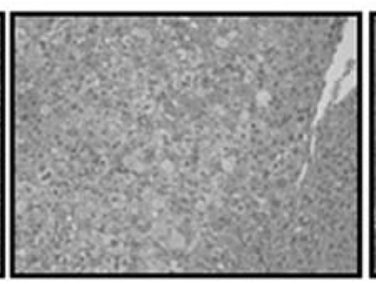

HCA

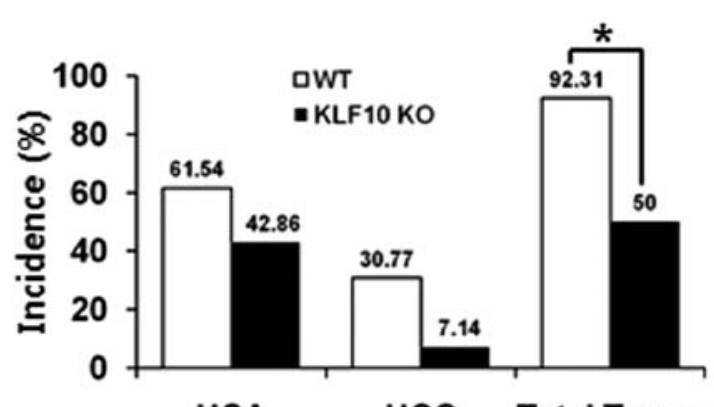

HCA

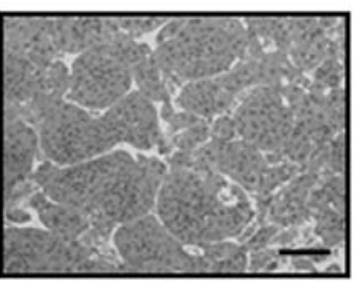

HCC

\section{8 weeks old}

Figure 2. Histopathological findings of the liver tissues. (A) H\&E staining of the normal liver tissues, heptic foci, HCA and HCC. Scale bar, 100 $\mu$ m. (B) Incidence of the HCA, HCC and total tumors. The incidence of HCA, HCC and total tumors was higher in the WT than the incidence in the KLF10 KO mice at both 28 and 38 weeks of age. The combined incidence of adenomas and carcinomas was significantly higher in the WT mice at 36 weeks after DEN treatment $(\mathrm{P}=0.021) .{ }^{*} \mathrm{P}<0.05$ by the Fisher's exact test. HCA, hepatocellular adenoma; HCC, hepatocellular carcinoma; WT, wild-type; DEN, diethylnitrosamine.

A

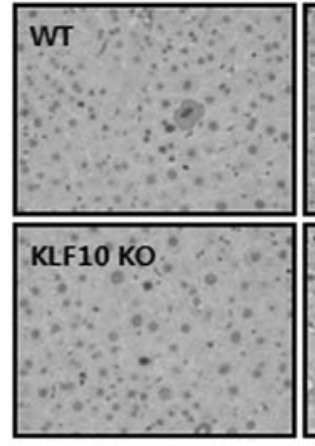

Normal
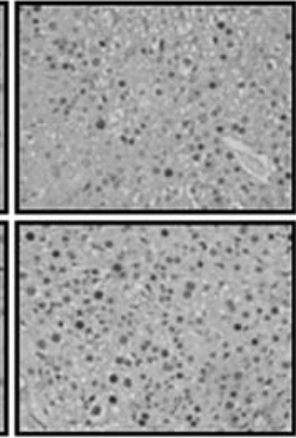

Hepatic foci

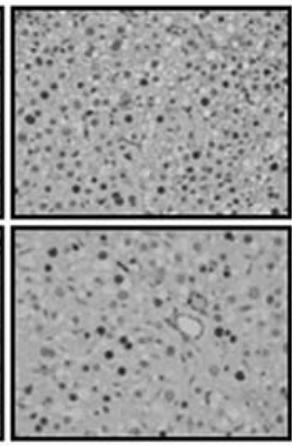

HCA

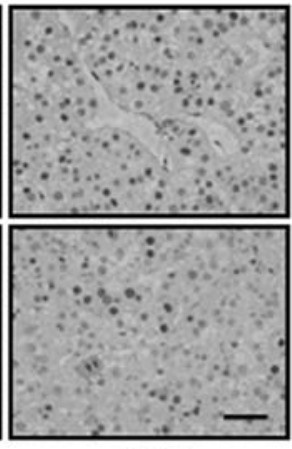

$\mathrm{HCC}$

B

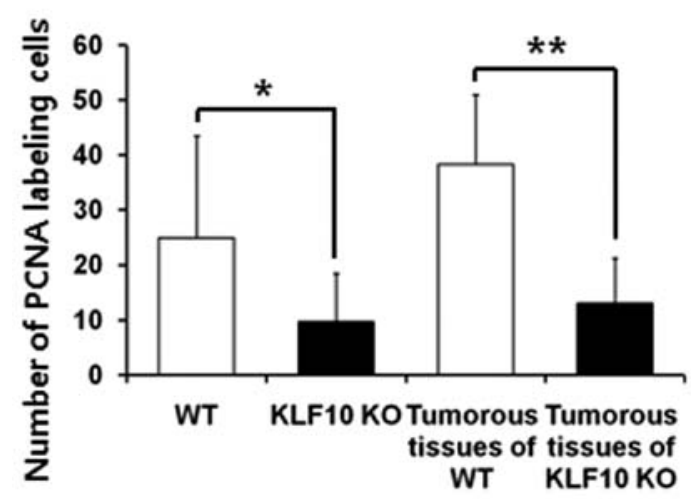

28 weeks old

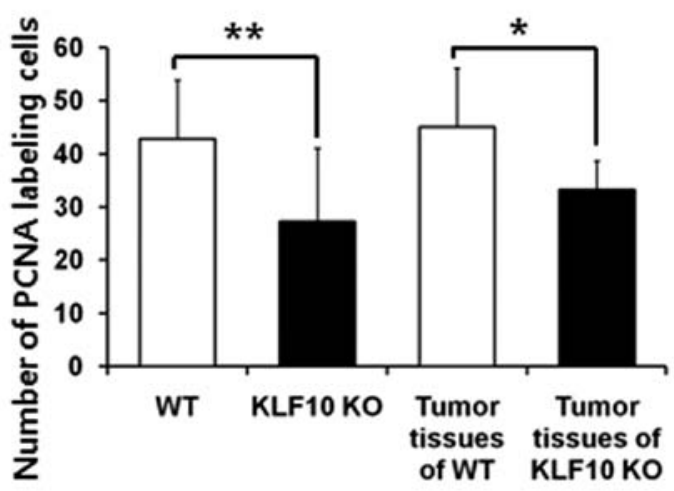

38 weeks old

Figure 3. Analyses of the proliferative potential of the liver cells. (A) PCNA stainings of the normal liver, hepatic foci, HCA and HCC. The number of PCNApositive nuclei was greater in the WT than that in the KLF10 KO mice. Scale bar, $50 \mu \mathrm{m}$. (B) PCNA labeling indices. The number of PCNA-positive cells was significantly higher in the WT than that in the KLF10 KO mice at both 28 and 38 weeks of age. When comparing only the tumor tissues including the preneoplastic hepatic foci, adenoma and hepatocellular carcinoma, the values were significantly higher in the WT mice at the age of 28 weeks. When comparing only the tumor tissues including the hepatocellular adenoma and carcinoma, the values were also significantly higher in the WT mice at the age of 38 weeks. ${ }^{*} \mathrm{P}<0.05$ and ${ }^{* *} \mathrm{P}<0.01$ by the unpaired Student's t-test. HCA, hepatocellular adenoma; HCC, hepatocellular carcinoma; WT, wild-type. 


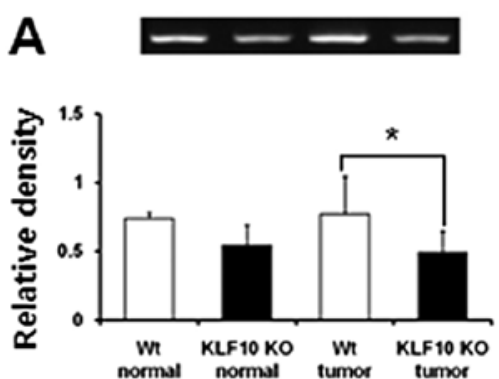

Smad2

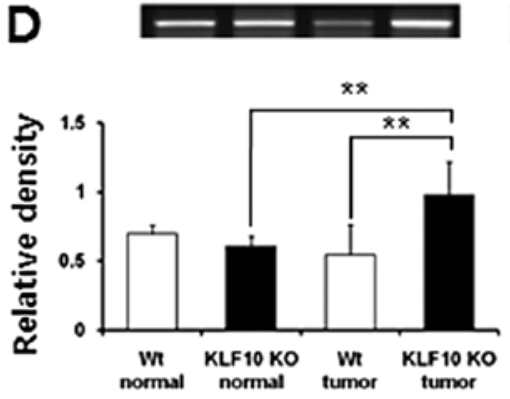

Smad3

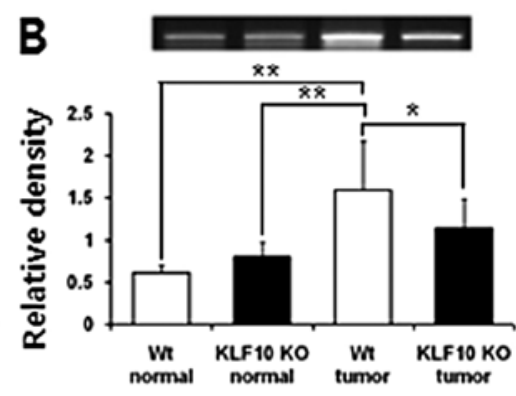

cMyc

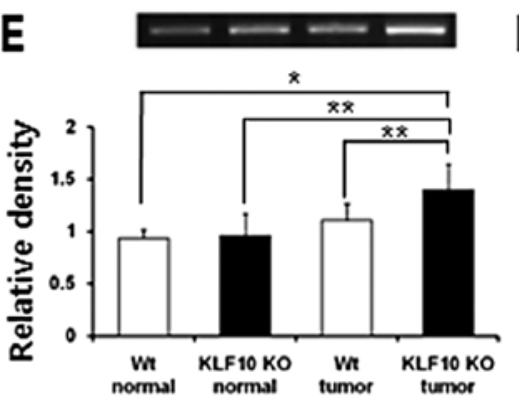

p15

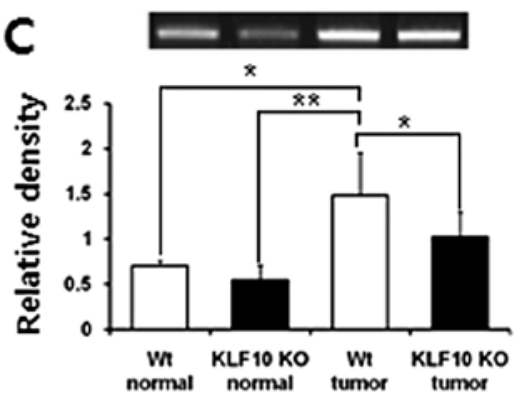

Cyclin D1

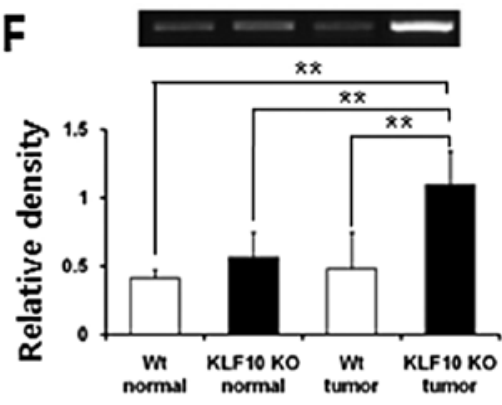

TGF- $\beta$ R1

Figure 4. Analyses of the mRNA expression levels of Smad2, cMyc, cyclin D1, Smad3, p15 and TGF- $\beta$ R1, which are involved in the TGF- $\beta /$ Smad pathway. (A-C) Smad2, cMyc and cyclin D1 were expressed significantly higher in the WT mice. (D and E) Smad3 and P15 were expressed significantly higher in the KLF10 KO mice. (F) TGF- $\beta$ RI was expressed significantly higher only in the KLF10 KO tumor group. $\beta$-actin was used as the housekeeping gene. ${ }^{*} \mathrm{P}<0.05$ and ${ }^{* *} \mathrm{P}<0.01$ by unpaired Student's t-test. TGF- $\beta$, transforming growth factor- $\beta$; WT, wild-type.

pattern was the most prominent feature (Fig. 2A). At the age of 28 weeks, the incidence of HCA in the KLF10 KO vs. the WT mice was $14.30(2 / 14)$ and $42.90 \%(6 / 14)$, respectively, but there were no malignant lesions observed in any mice. At the age of 38 weeks, the incidence of HCA, HCC and total tumors was $42.86(6 / 14), 7.14(1 / 14)$ and $50.00 \%(7 / 14)$ in the KLF10 KO mice and $61.54(8 / 13), 30.77(4 / 13)$ and $92.31 \%(12 / 13)$ in the WT mice, respectively. The tumor incidence was significantly higher in the WT than that in the KLF10 KO mice (Fig. 2B; $\mathrm{P}=0.021$ by Fisher's exact test).

Comparison of the cellular proliferative potential of liver tissues in the WT and KLF1O KO mice. The immunohistochemical staining for PCNA was assessed to analyze the proliferative potential of hepatocytes. The number of PCNApositive nuclei was counted in an area of $0.1 \mathrm{~mm}^{2}$ under a light microscope. The PCNA labeling index was significantly higher in the WT than that in the KLF10 KO mice. At 26 weeks after DEN treatment, the mean \pm SD values of the WT and KLF10 KO mice were $24.9 \pm 18.6$ and $9.64 \pm 8.83$, respectively $(\mathrm{P}=0.010)$. When comparing only tumorous tissues including pre-neoplastic hepatic foci, the values were $38.3 \pm 12.7$ and $13.1 \pm 8.19$, respectively $(\mathrm{P}=0.0023)$. At 36 weeks after $\mathrm{DEN}$ administration, the mean \pm SD values of the WT and KLF10 KO mice were $42.8 \pm 11.2$ and $27.2 \pm 13.8$, respectively $(\mathrm{P}=0.0060)$. When comparing only tumorous tissues excluding hepatic foci tissues, the values were $45.1 \pm 11.1$ and $33.2 \pm 5.40$, respectively $(\mathrm{P}=0.041)$ (Fig. 3).

Analyses of expression of genes involved in the TGF- $\beta / S m a d$ signaling pathway. To investigate the mRNA transcription levels of the genes (Smad2, Smad3, Smad4, Smad7, p15, p21, TGF- $\beta$ RI, TGF- $\beta$ RII, cMyc and cyclin D1) involved in the TGF- $\beta$ /Smad pathway, RT-PCR analyses were performed. The mRNA levels of Smad2, one of the KLF10 target genes, were higher in the WT than levels in the KLF10 KO mice and a significant difference was obtained in the tumor tissues. The levels of Smad3 and p15, antiproliferative genes, were significantly higher in the tumor tissues of the KLF10 KO than levels in the tumor tissues of the WT mice. TGF- $\beta$ RI, which is involved in the early stage of the TGF- $\beta$ pathway and may potentially inhibit cellular proliferation (10), was expressed prominently in the KLF10 KO tumor tissues when compared with the other tissues. In contrast, cMyc and cyclin D1 genes associated with cellular multiplication showed significantly higher expression in the WT tumors than levels in the other tissues (Fig. 4). Other genes, Smad2, Smad4, Smad7, p21 and TGF- $\beta$ RII, did not have any significant difference in mRNA levels (data not shown).

Western blot analysis of proteins involved in the TGF- $\beta / S m a d$ signaling pathway. To examine the expression levels of the TGF- $\beta$ pathway target or associated proteins [Smad2, Smad3, Smad4, Smad7, phospho-Smad2 (p-Smad2), phospho-Smad3 (p-Smad3; ser433/435), p15, p21, p27, TGF- $\beta 1$, TGF- $\beta$ RI, TGF- $\beta$ RII and cyclin D1], western blot analysis of each protein was assessed and normalized to the $\beta$-actin level. Similar to the quantitative RT-PCR results, the protein levels of Smad2 were significantly higher in the WT than levels in the KLF10 KO tissues. Likewise, Smad3 and p15 levels were significantly higher in the KLF10 KO than levels in the WT tumor and the normal KLF10 KO tissues. The TGF- $\beta$ RI protein level 


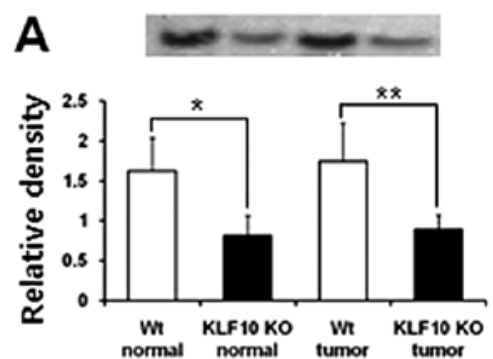

Smad2

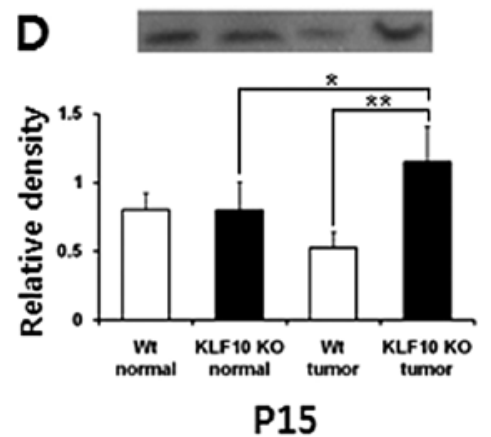

G

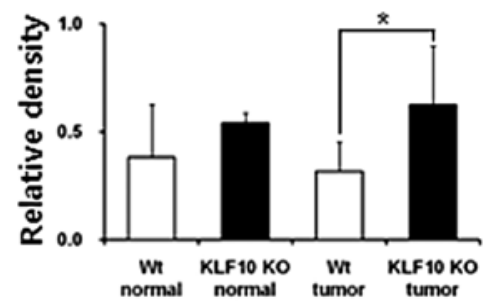

pSmad3

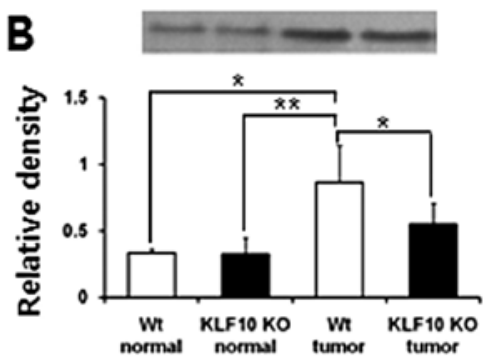

Cyclin D1

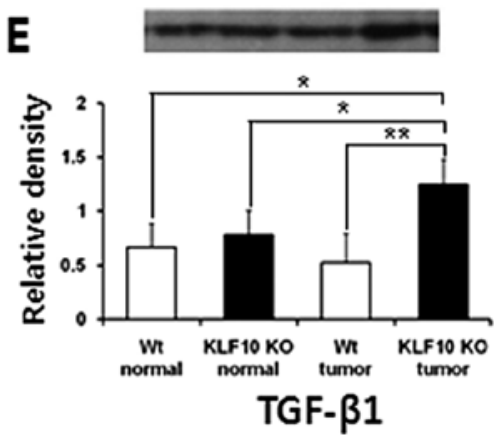

H

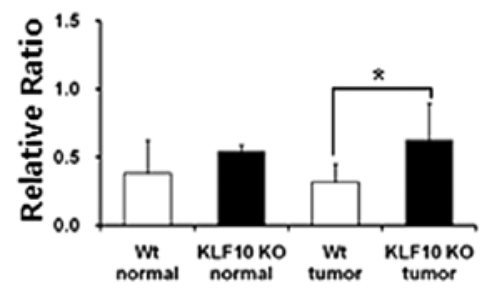

p-Smad3/Smad3

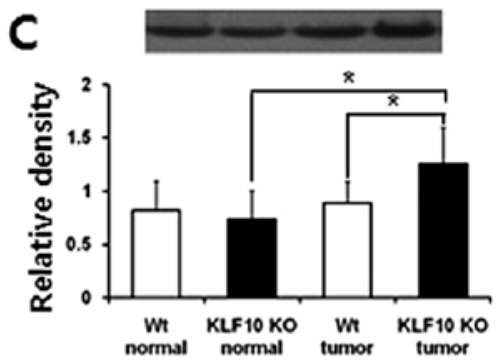

Smad3

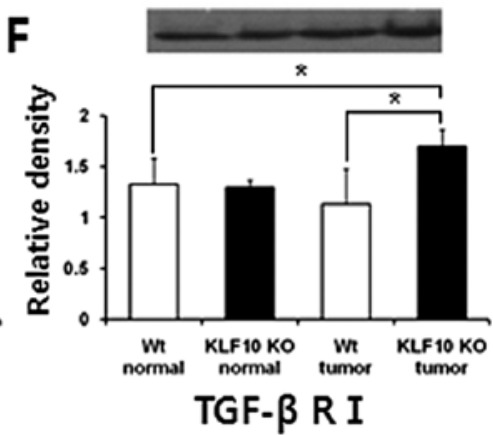

Figure 5. Quantification of the protein expression levels of Smad2, cyclin D1, Smad3, p15, TGF- $\beta 1$, TGF- $\beta$ R1 and pSmad3, which are involved in the TGF- $\beta$ /Smad pathway. (A and B) Smad 2 and cyclin D1 were expressed significantly higher in the WT groups. (C-F) Smad3, P15, TGF- $\beta 1$ and TGF- $\beta$ RI were expressed significantly higher in the KLF10 KO mice. (G) pSmad3 expression and (H) Smad3 activation were significantly higher only in the KLF10 KO tumor group. The band intensities were quantified and normalized to the $\beta$-actin level. ${ }^{*} \mathrm{P}<0.05$ and ${ }^{* *} \mathrm{P}<0.01$ by the unpaired Student's t-test. TGF- $\beta$, transforming growth factor- $\beta$; WT, wild-type.

was also significantly higher in the KLF10 KO than levels in the WT tumor and normal tissues. TGF- $\beta 1$, the initiator of the TGF- $\beta$ pathway and one of the KLF10 target genes, was higher in the KLF10 KO tumors than that in the other tissues. In contrast, the cyclin D1 level was significantly higher in the WT tumors than this level in the other tissues (Fig. 5). Other proteins, Smad4, Smad7, p-Smad2, p21, p27 and TGF- $\beta$ RII, did not have any meaningful difference in protein levels (data not shown). Additional analysis of p-Smad3, the activated form of Smad3, was performed, and the expression level in the KLF10 KO tumors was more dominant than that in the WT tumors $(\mathrm{P}=0.042)$ (Fig. 5C). The $\mathrm{p}-\mathrm{Smad} 3 / \mathrm{Smad} 3$ ratio used to analyze the degree of Smad3 activation was also significantly higher in the KLF10 KO, than this ratio in the WT tumor tissues (Fig. 5H).

\section{Discussion}

KLF10, first observed in 1995, is known to induce upregulation of TGF- $\beta$ target genes by enhancement of R-Smad and co-Smad activation. It may also enhance TGF- $\beta$ signaling transduction by induction of the expression of several genes involved in TGF- $\beta$ signaling, Smad 2 and TGF- $\beta 1(21,27,28)$. TGF- $\beta$ may regulate many biological processes in most cells, and cellular responses to TGF- $\beta$ are different under various conditions $(11,12)$. The actual functions of TGF- $\beta$ with KLF10 in various pathophysiological conditions still remain unclear $(13,20,24)$. Particularly, there have not been any in vivo studies to elucidate the roles of KLF10 in the liver. In the present study, functions of the KLF10 gene in chemically induced hepatic tumorigenesis were investigated using KLF10 KO mice. The mouse model of DEN-induced HCC, which was also employed in the present study, has been extensively used for liver carcinogenesis studies since it is genetically and histologically similar to the human one $(29,30)$.

In the necropsy and histopathological results, KLF10 KO mice exhibited a lower incidence of tumors (Figs. 1C and 2B). Additionally, PCNA labeling indices were significantly higher in the WT than indices in the KLF10 KO mice (Fig. 3B). Considering these results, KLF10 KO mice may benefit from antiproliferative activity following chemically induced hepatic tumorigenesis. To elucidate the causes behind the increased 
antiproliferative effects in KLF10 KO mice, the expression levels of genes involved in the TGF- $\beta /$ Smad pathway were examined by quantitative RT-PCR. The expression level of the Smad2 gene, one of the KLF10 target gene, was higher in the WT than that in the KLF10 KO mice, and the significant difference was obtained between the tumor tissues of the WT mice and those of the KLF10 KO (Fig. 4A). This may be caused by malfunction of the KLF10 gene in KO mice, and the difference was intensified in the tumor tissues. However, Smad7 and p21, previously also reported as target genes of KLF10, did not show any significant differences. Smad4 and TGF- $\beta$ RII, mediators of TGF- $\beta$ signaling, did not have any significant differences either (data not shown). This may indicate that KLF10 ablation did not affect the expression of the genes mentioned above in hepatic carcinogenesis, except for Smad2. In contrast, mRNA levels of Smad3, TGF- $\beta$ RI and p15 were increased in the tumor tissues of the KLF10 KO mice, and cMyc, cyclin D1 were expressed prominently in the tumor tissues of the WT mice (Fig. 4B-F). In the western blot analysis, similar results were obtained, and TGF- $\beta 1$ was expressed significantly higher in the tumor tissues of the KLF10 KO mice than in other tissues. In addition, the $\mathrm{p}$-Smad3/Smad3 ratio was significantly higher in the tumor tissues of the KLF10 KO mice (Fig. 5). Considering these results, the KLF10 null condition induced abundant expression of TGF- $\beta$ R1, TGF- $\beta 1$, Smad 3 and increased activation of Smad3. It reinforced the TGF- $\beta /$ Smad pathway in chemically induced carcinogenesis. As a result, p15, one of the positively regulated target genes of the TGF- $\beta$ pathway, was increased in the KLF10-deficient mice and it suppressed transcription and function of proliferation-related genes, cMyc and cyclin D1. The activated Smad complex may also directly suppress transcription of the cMyc gene. Thus, lack of KLF10 suppressed cellular proliferation of hepatocytes during liver tumorigenesis by reinforcement of the TGF- $\beta /$ Smad pathway.

TGF- $\beta$ induces a great variety of cellular responses dependent on the type of cells and the microenvironments $(15,18,31)$. KLF10, one of the early target genes of TGF- $\beta$, is also involved in various biological processes and diseases. Most previous studies have shown that the effects of KLF10 were similar to those of TGF- $\beta$, including antiproliferation; however, this information remains insufficient and fractionary (21,32-34). There is one study that examined the roles of KLF10 in liver cancer and it revealed that upregulation of KLF10 in an HCC cell line may induce inhibition of cellular proliferation (35). However, dissimilar to the cell culture environment, the KLF10 null condition in the liver of a living organism may induce various other responses, as in the present study, due to differences in the composition of the cells, the architecture of the tissue and the microenvironment.

Likewise, various biological responses may be induced in the same knockout mouse. For example, tumorigenesis of the lymphoid system was accelerated, while hepatic carcinogenesis was suppressed in ataxia telangiectasia-mutated gene (ATM) KO mice. This may be caused by a dissimilar process of malignancy derived from differences in the cell type that participate in tumorigenesis and the microenvironment (36-38). One study concerning tumorigenesis using KLF10 KO mice showed that the incidence of skin cancer was increased in KLF10 KO mice (26). As mentioned above, diverse responses may be induced in tumorigenesis according to the type of tissues, although the same genetically engineered mice were used. Therefore, results showing tumor suppressor activity can be obtained in liver carcinogenesis study using KLF10 KO mice, although previous studies were mainly focused on the tumor-suppressor effects of KLF10.

TGF- $\beta$ may activate Ras, Rho, TAK1 and PP2A except for Smad proteins. In addition, KLF10 may be induced by bone morphogenetic proteins, estrogen and epidermal growth factor except for TGF- $\beta(17,19,39)$. Furthermore, KLF11 was recently found to exhibit similar effects as $\operatorname{KLF} 10(21,40)$. Considering these studies, some compensatory mechanisms for the KLF10 null condition may exist in the non-Smad TGF- $\beta$ pathways or KLF10 signaling. Thus, to elucidate the exact mechanisms in KLF10 KO mice, additional analyses of intracellular signaling pathways (except for Smads) are needed, and also the effects of other inducers of KLF10 (except for TGF- $\beta$ ) are warranted. In addition, to confirm the antiproliferative effect of the KLF10 null condition, in vivo analysis concerning the KLF10 blockade using WT mice is also needed.

In conclusion, the KLF10 KO mice exhibited a lower tumor incidence and cellular proliferation than the WT mice following DEN-induced liver tumorigenesis. Although the expression of the $\mathrm{Smad} 2$ gene was decreased in the hepatic tissues of the KLF10 KO mice, the TGF- $\beta /$ Smad signaling pathway was reinforced by upregulation of Smad3, TGF- $\beta 1$, TGF- $\beta$ R1 and increased activation of Smad3. This induced increased expression of p15 and the suppression of proliferation-related genes, cMyc and cyclin D1. Based on the results, KLF10 KO mice may benefit from antiproliferative activities following liver tumorigenesis by enhancement of the TGF- $\beta$ pathway. This study provides the first observation concerning the mito-inhibitory effects in liver cells using KLF10 KO mice. However, the exact mechanisms and effects of the tumor-suppressor activities in KLF10 KO mice require further elucidation. To confirm these mechanisms, additional analysis of the non-Smad TGF- $\beta$ pathway and in vivo examinations using WT mice with KLF10 blocking agents are needed.

\section{Acknowledgements}

This study was supported by Konkuk University in 2014 .

\section{References}

1. Ferlay J, Shin HR, Bray F, Forman D, Mathers C and Parkin DM: Estimates of worldwide burden of cancer in 2008: GLOBOCAN 2008. Int J Cancer 127: 2893-2917, 2010.

2. Jemal A, Bray F, Center MM, Ferlay J, Ward E and Forman D: Global cancer statistics. CA Cancer J Clin 61: 69-90, 2011.

3. Shin HR, Carlos MC and Varghese C: Cancer control in the Asia Pacific region: current status and concerns. Jpn J Clin Oncol 42: 867-881, 2012.

4. Maluccio $\mathrm{M}$ and Covey A: Recent progress in understanding, diagnosing, and treating hepatocellular carcinoma. CA Cancer J Clin 62: 394-399, 2012

5. Villanueva A, Newell P, Chiang DY, Friedman SL and Llovet JM: Genomics and signaling pathways in hepatocellular carcinoma. Semin Liver Dis 27: 55-76, 2007

6. Farazi PA and DePinho RA: Hepatocellular carcinoma pathogenesis: from genes to environment. Nat Rev Cancer 6: 674-687, 2006.

7. Sia D and Villanueva A: Signaling pathways in hepatocellular carcinoma. Oncology 81: 18-23, 2011. 
8. Villanueva A, Chiang DY, Newell P, et al: Pivotal role of mTOR signaling in hepatocellular carcinoma. Gastroenterology 135: 1972-1983, 2008.

9. Kaminska B, Wesolowska A and Danilkiewicz M: TGF beta signaling and its role in tumour pathogenesis. Acta Biochim Pol 52: 329-337, 2005.

10. Inman GJ: Switching TGF- $\beta$ from a tumor suppressor to a tumor promoter. Curr Opin Genet Dev 21: 93-99, 2011.

11. Breitkopf K, Weng H and Dooley S: TGF- $\beta /$ Smad-signaling in liver cells: Target genes and inhibitors of two parallel pathways. Signal Transduction 6: 329-337, 2006.

12. Achyut BR and Yang L: Transforming growth factor- $\beta$ in the gastrointestinal and hepatic tumor microenvironment. Gastroenterology 141: 1167-1178, 2011.

13. Massagué J: TGFbeta in cancer. Cell 134: 215-230, 2008.

14. Fausto N, Campbell JS and Riehle KJ: Liver regeneration. Hepatology 43: S45-S53, 2006.

15. Itoh $S$ and ten Dijke P: Negative regulation of TGF- $\beta$ receptor/Smad signal transduction. Curr Opin Cell Biol 19: 176-184, 2007.

16. Ellenrieder V: TGFbeta regulated gene expression by Smads and Sp1/KLF-like transcription factors in cancer. Anticancer Res 28: $1531-1539,2008$.

17. Kang JS, Liu C and Derynck R: New regulatory mechanisms of TGF-beta receptor function. Trends Cell Biol 19: 385-394, 2009.

18. Derynck R, Akhurst RJ and Balmain A: TGF-beta signaling in tumor suppression and cancer progression. Nat Genet 29 : $117-129,2001$

19. Moustakas A and Heldin CH: Non-Smad TGF-beta signals. J Cell Sci 118: 3573-3584, 2005.

20. Subramaniam M, Hawse JR, Rajamannan NM, Ingle JN and Spelsberg TC: Functional role of KLF10 in multiple disease processes. Biofactors 36: 8-18, 2010.

21. Spittau B and Krieglstein K: Klf10 and Klf11 as mediators of TGF-beta superfamily signaling. Cell Tissue Res 347: 65-72, 2012.

22. Subramaniam M, Harris SA, Oursler MA, Rasmussen K and Riggs BL: Identification of a novel TGF-beta-regulated gene encoding a putative zinc finger protein in human osteoblasts. Nucleic Acids Res 23: 4907-4912, 1995.

23. Cook T, Gebelein B, Belal M, Mesa K and Urrutia R: Three conserved transcriptional repressor domains are a defining feature of the TIEG subfamily of Sp1-like zinc finger proteins. J Biol Chem 274: 29500-29504, 1999

24. Johnsen SA, Subramaniam M, Janknecht R and Spelsberg TC: TGF-beta inducible early gene enhances TGFbeta/Smad-dependent transcriptional responses. Oncogene 21: 5783-5790, 2002.

25. Subramaniam M, Hawse JR, Johnsen SA and Spelsberg TC: Role of TIEG1 in biological processes and disease states. J Cell Biochem 102: 539-548, 2007.
26. Song KD, Kim DJ, Lee JE, Yun CH and Lee WK: KLF10, transforming growth factor- $\beta$-inducible early gene 1 , acts as a tumor suppressor. Biochem Biophys Res Commun 419: 388-394, 2012.

27. Dooley S, Weng H and Mertens PR: Hypotheses on the role of transforming growth factor-beta in the onset and progression of hepatocellular carcinoma. Dig Dis 27: 93-101, 2009.

28. Buenemann CL, Willy C, Buchmann A, Schmiechen A and Schwarz M: Transforming growth factor-beta1-induced Smad signaling, cell-cycle arrest and apoptosis in hepatoma cells. Carcinogenesis 22: 447-452, 2001.

29. Newell P, Villanueva A, Friedman SL, Koike K and Llovet JM Experimental models of hepatocellular carcinoma. J Hepatol 48: 858-879, 2008.

30. Bakiri L and Wagner EF: Mouse models for liver cancer. Mol Oncol 7: 206-223, 2013

31. Heldin $\mathrm{CH}$ and Miyazono $\mathrm{K}$ : Transforming growth factor-beta. An interesting candidate for clinical use. Lakartidningen 92: 1569-1572, 1995 (In Swedish).

32. Bensamoun SF, Hawse JR, Subramaniam M, et al: TGF-beta inducible early gene-1 knockout mice display defects in bone strength and microarchitecture. Bone 39: 1244-1251, 2006.

33. Tsubone T, Moran SL, Subramaniam M, Amadio PC, Spelsberg TC and An KN: Effect of TGF-beta inducible early gene deficiency on flexor tendon healing. J Orthop Res 24: 569-575, 2006.

34. Bos JM, Subramaniam M, Hawse JR, et al: TGF $\beta$-inducible early gene-1 (TIEG1) mutations in hypertrophic cardiomyopathy. J Cell Biochem 113: 1896-1903, 2012.

35. Jiang L, Lai YK, Zhang JF, et al: Transactivation of the TIEG confers growth inhibition of transforming growth factor- $\beta$ susceptible hepatocellular carcinoma cells. World J Gastroentero 18: 2035-2042, 2012

36. Reliene R and Schiestl RH: Antioxidants suppress lymphoma and increase longevity in Atm-deficient mice. J Nutr 137: S229-S232, 2007.

37. Teoh N, Pyakurel P, Dan YY, et al: Induction of p53 renders ATMdeficient mice refractory to hepatocarcinogenesis. Gastroenterology 138: 1155-1165, 2010.

38. Stracker TH, Roig I, Knobel PA and Marjanović M: The ATM signaling network in development and disease. Front Genet 4: 37, 2013.

39. Derynck R and Zhang YE: Smad-dependent and Smad independent pathways in TGF-beta family signalling. Nature 425 : 577-584, 2003.

40. Gohla G, Krieglstein K and Spittau B: Tieg3/Klf11 induces apoptosis in OLI-neu cells and enhances the TGF-beta signaling pathway by transcriptional repression of Smad7. J Cell Biochem 104: 850-861, 2008. 